\title{
Ultra-Wide Field of View Lens-Let Array for Efficient Solar Collection
}

\author{
Rakan E. Alsaigh, ${ }^{1}$ Ralf Bauer, ${ }^{2}$ and Martin P.J. Lavery ${ }^{1}$ \\ 'School of Engineering, University of Glasgow, Glasgow, UK \\ Department of Electronic and Electrical Engineering, University of Strathclyde, Glasgow, UK \\ Authors e-mail address: r.alsaigh.1@research.gla.ac.uk,ralf.bauer@strath.ac.ukand martin.lavery@glasgow.ac.uk
}

\begin{abstract}
Efficient solar collection over a full-day is a leading challenge for photovoltaic power generation. We present a novel multi-layer lens-let array that increases the daily collection efficiency of standard panels by a factor of 2.32 .
\end{abstract}

(C) 2019 R. E. Alsaigh, R. Bauer, M. P.J. Lavery

OCIS codes: $350.0350,350.6050$. For codes, see http://www.osapublishing.org/submit/ocis/

Photovoltaics (PV) continue to be at the centre of renewable technologies with a promising potential to powering many cutting-edge applications, including future generation autonomous platforms, portable electronic systems and smart communication networks. New developments in the fields of dynamic control [1], artificial intelligence [2] and smart telecom networks [3] are major drivers for energy consumption requiring sustainable power. The universal deployment of solar technologies is still constrained by the ability to efficiently collect sunlight from a wide range of angles and at different alignment scenarios. Traditionally, the angle-dependent efficiency constraint has been tackled through the use of mechanical tracking systems that are expensive, require continual maintenance and the use of sensors to create automatic systems. Novel optical elements are an interesting route to provide passive wide field of view optical collectors for solar power systems. However, given the maturity of current manufacturing techniques, such systems compatibility with standard solar technology will be a necessity.

We will present a novel lens-let array design that allows for ultra-wide angle solar collection of \pm 80 degrees, can be incorporated into any standard and commercial solar panels and increases their daily production of open-circuit voltage by a multiplicative factor of 2.32. Our optical design consists of two short focal-length hemispheric lens-let arrays arranged with their curved faces opposite to each other, see Fig.1(a), resulting in an element with a field-ofview (FOV) of 80 degrees [4]. This arrangement in the form of an array leads the entering rays, at high or low incident angle, to either be collected by opposite lens or other adjacent lenses in the second array, as illustrated in Fig.1(a), creating a wide lens distribution and minimum ray loss. This allows for efficient capture of incoming light and hence a significant increase in the overall solar-to-electricity conversion.

(a)
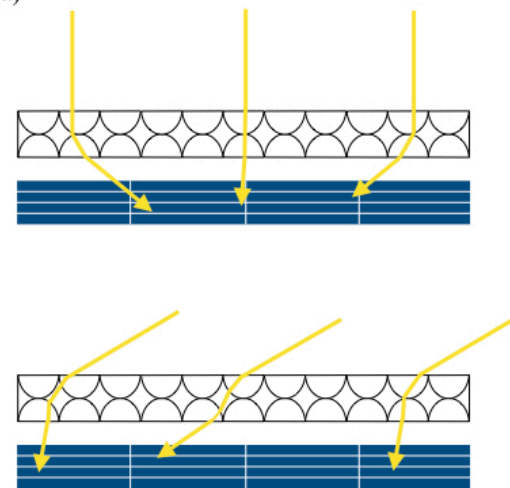

(b)

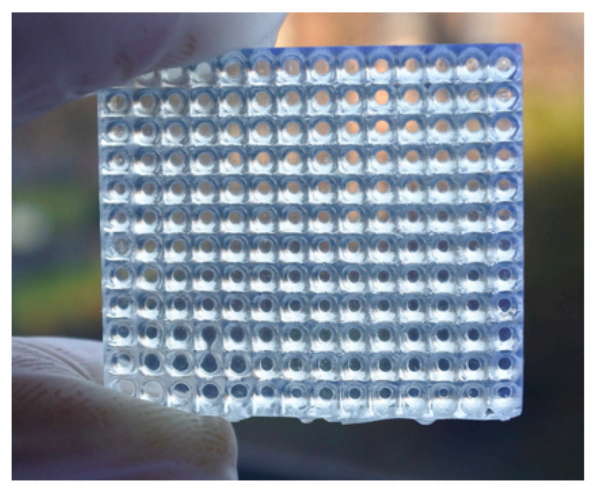

Fig. 1. Ultra-wide field of view optical element. (a) Illustration of our multi-layer lens-let arrays that forms the optical elements, with simulated rays with incident angles of 0 degree (top) and 60 degrees (bottom). (b) Image of our 3D-printed optical prototype.

The fabrication of our lens-let array is achieved through the use of stereolithographic 3D-printing technologies that can rapidly prototype transparent components. To transform the 3D-printed lens into optical quality components, 
each lens-let arrays undergo multiple post-processing steps that reduces their surface roughness and enhances their optical transparency. Our process has shown an improved optical loss in contrast to the losses indicated by other research teams [5, 6]. Two separate $29.05 \times 24.30 \mathrm{~mm}$ lens-let arrays were prototyped and then bonded together to form the multi-layer optical element, as shown in Fig. 1(b). Beside this prototype, our design can easily be manufactured using standard manufacturing techniques of optics and tailored to fit on top of any commercial or future PV panels. The integration of our 3D-printed optical element with an "off-the-shelf" Amorphous Silicon solar panel (Sanyo, AM-1815CA) demonstrated significant multiplicative gains in open-circuit voltage $\left(\mathrm{V}_{\mathrm{oc}}\right)$ over its flat counterpart by a factor of 1.04 to 2.32, depending on the panel's latitudinal tilt angle as shown in Fig. 2(a) and (b).

(a)

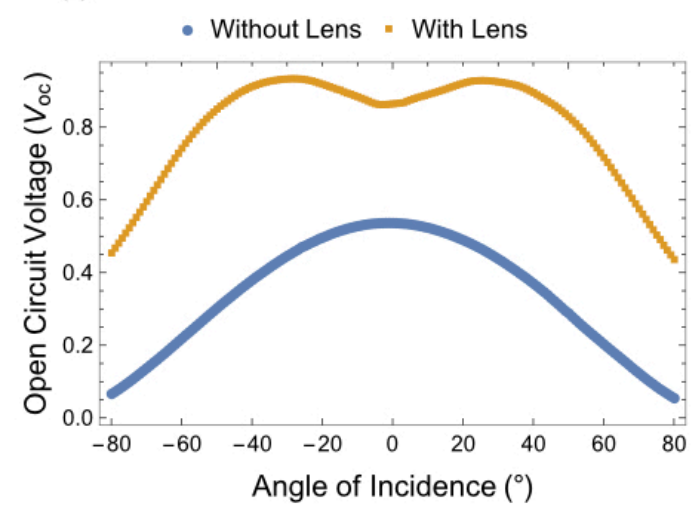

(b)

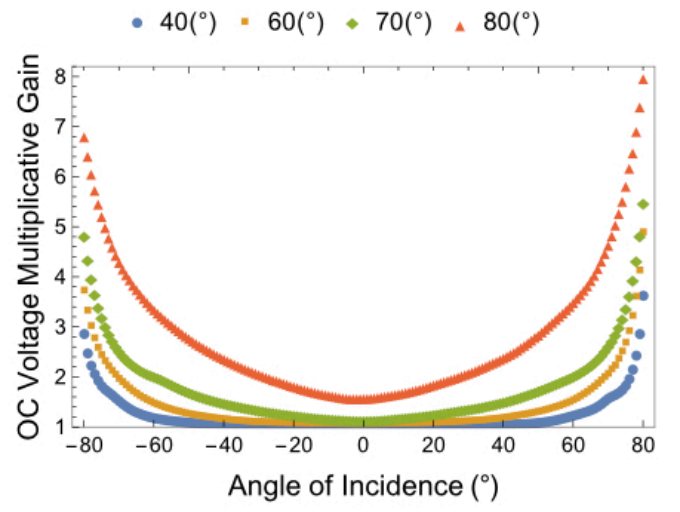
element into the same PV panel at longitudinal tilt angle of $80^{\circ}$. (b) Multiplicative gains in open circuit voltage for the element-integrated PV panel over its flat counterpart at longitudinal tilt angle of $40^{\circ}, 60^{\circ}, 70^{\circ}$ and $80^{\circ}$.

We developed an optical simulation for our design to predict the optical power delivered to the solar panel surface. Through tracing the light entering one lens-let on the top layer, we find the majority of optical power is couple into five neighbouring lens-lets on the second layer of the element. Further, we simulate the ray-direction of the transmitted light from our optical element over input incident angles of 0-80 degree, confirming the majority of input rays are redirected by our element to match the field of view of the solar panel. Based on this simulation, we will discuss the role of miniaturised lens-lets and array distribution in the enhancement of the overall collection efficiency of our proposed system.

We will present the latest characterization results of our optical element, including simulations, fabrication procedures and results from incorporating it with "off-the-shelf" solar panel for solar energy collection. The simulation results will show the predicted angular deflection of the rays and the overall optical power outputting our system. Further, our experimental measurements will present the total power produced by our system at various latitudinal tilt angles. Our lens-let array could provide an efficient method to power system that don't have a fix relationship with the sun, such as: electric vehicles, aircrafts and drones.

\section{References}

[1]. Robert J. Griffin, Georg Wiedebach, Sylvain Bertrand, Alexander Leonessa and Jerry Pratt, "Walking stabilization using step timing and location adjustment on the humanoid robot, Atlas," 2017 IEEE/RSJ International Conference on Intelligent Robots and Systems (IROS), 667-673 (2017).

[2]. Sergey Levine, Peter Pastor, Alex Krizhevsky, Julian Ibarz and Deirdre Quillen, "Learning hand-eye coordination for robotic grasping with deep learning and large-scale data collection," The International Journal of Robotics Research 37(4-5), 421-436 (2018).

[3]. Martin P. J. Lavery, Mojtaba Mansour Abadi, Ralf Bauer, Gilberto Brambilla, Ling Cheng, Mitchell A. Cox, Angela Dudley, Andrew D. Ellis, Nicolas K. Fontaine, Anthony E. Kelly, Christoph Marquardt, Selaelo Matlhane, Bienvenu Ndagano, Francesco Petruccione, Radan Slavík, Filippo Romanato, Carmelo Rosales-Guzmán, Filippus S. Roux, Kobus Roux, Jian Wang and Andrew Forbes, “Tackling Africa’s digital divide” Nature Photonics 12, 249-252 (2018).

[4]. M. Rezaei, I. H. Nia, A. Bonakdar, H. Mohseni, "Simple telecentric submillimeter lens with near-diffraction-limited performance across an 80 degree field of view," App. Opt. 55, 8752- 8756 (2016).

[5]. J. S. Price, X. Sheng, B. M. Meulblok, J. A. Rogers, N. C. Giebink, "Wide-angle planar microtracking for quasi-static microcell concentrating photovoltaics," Nat. Commun. 6, 6223 (2015).

[6]. J. Gawedzinski, M. E. Pawlowski, T. S. Tkaczyk, "Quantitative evaluation of performance of 3D printed lenses,” Opt. Eng. 56, 084110 (2017). 